\title{
THE LEG TENDONS OF INSECTS
}

\author{
PROFESSOR C. W. WOODWORTH \\ University of CALIFornia
}

WHILE perhaps known to working morphologists, the fact that the leg tendons are cuticular invaginations, and therefore subject to replacement at each molt, does not appear to have attracted the attention of any of the writers of text-books, and as far as the writer of this article is aware, has not been published at all.

The three best developed tendons are the two operating the knee joint and the one that flexes the claws. These three are almost invariably present, though one or the other may be very short, or present only as a cuticular thickening.

These structures are very easy to study in small insects. I have found aphids the most satisfactory subjects. The legs of most species are transparent enough to show the structures well when mounted whole, and the exuviæ are especially satisfactory objects. They may also be obtained in such abundance that one can mount large series of specimens, thus obtaining mounts showing the legs from almost any desired point of view.

The knee joints provide for the largest amount of motion of any of the joints of the leg, and this motion is all maintained in one plane by the development of two bearing points, making a hinge. The end of the tibia is small enough to telescope within the femur but for these articular processes. They consist of a process projecting inwardly on either side of the rim of the femur, as shown in Fig. 1, $A$ and $B$, and corresponding with these femoral processes there are slight outwardly projecting processes from the margin of the thickened rim of the tibia. The articular membrane at these points prevents the displacement of the processes. 
The whole dorsal end of the tibia, including these processes, is very largely hardened and thickened and marked off from the body of the tibia by a deeply infolded ridge. Most of this thickened portion is within the end

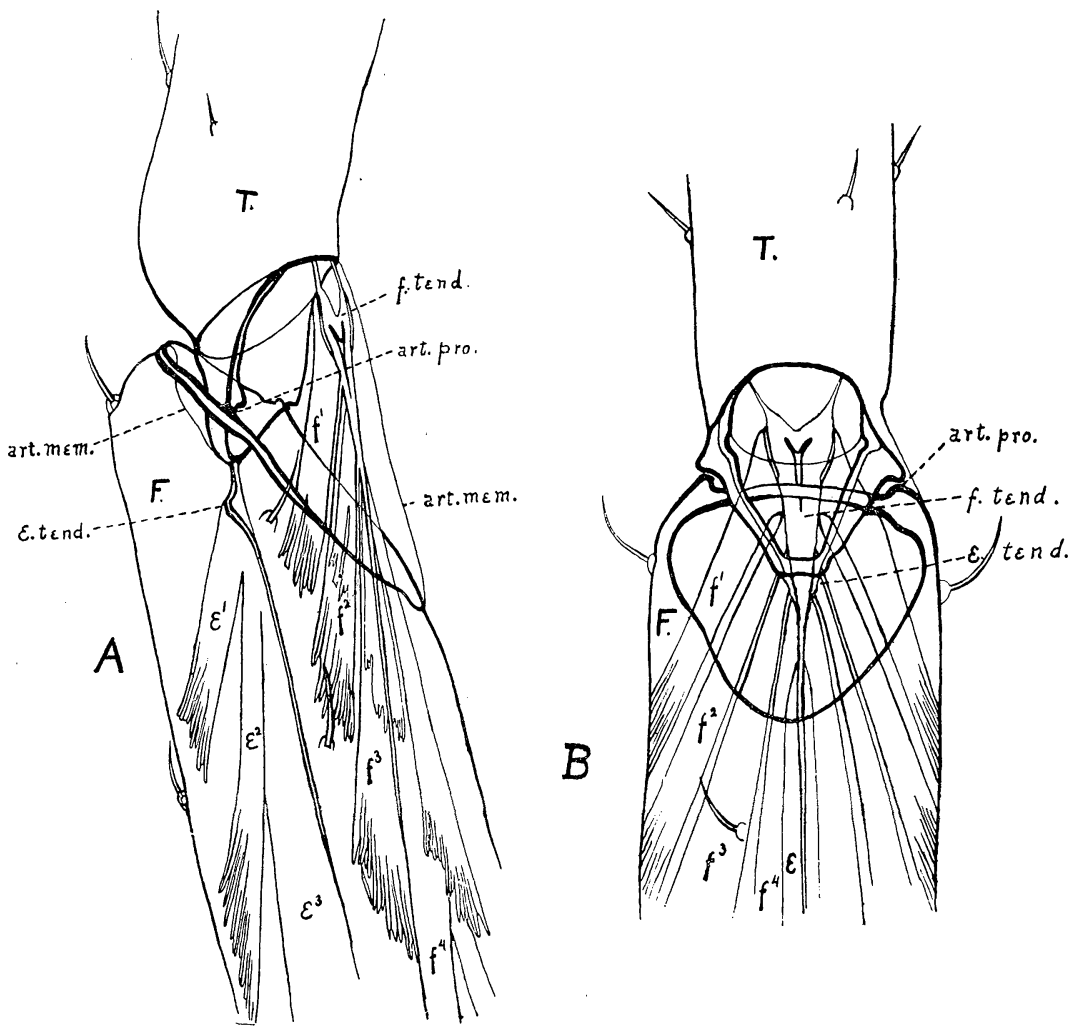

FIG. 1. The knee joint of Aphis brassick. A, side view; $B$, viewed from beneath; $T$, tibia; $F$, femur ; $a r^{\bullet}$.pro, articular process ; e.tend, extensor tendon; $e^{1} c^{2} c^{3}$, extensor muscles; f.tend, flexor tendon; $f^{1} f^{2} f^{3} f^{4}$, flexor muscles ; art.mem, articular membrane.

of the femur when the leg is fully extended, but is all exposed when the leg is at extreme flexion. An articular membrane connects the extreme edges of femur and tibia, as shown in Fig. 1, $A$.

Beneath, the hard parts of both femur and tibia are deeply emarginated, exposing a broad articular membrane. When in extreme flexion the rims of tibia and 
femur almost touch, and the articular membrane is drawn deep into the femoral cavity. The tendons find their attachment to the outer and inner sides of the rim of the tibia and, extending into the cavity of the femur, serve for the attachment of a series of muscles, as shown in Fig. 1, $A$ and $B$.

The flexor tendon in the earlier stages is only a $V$-shaped thickening of the articular membrane, but later the point of the $\mathrm{V}$ extends deeply as an internal pocket for the attachment of muscles reaching nearly to the base of the femur. There are two sets of muscles attached to this tendon, extending obliquely to the right and left sides of the femur. The first of these, $f^{1}$, in the figures lying at about $45^{\circ}$ to the long axis of the femur, and the others marked $f^{2}, f^{3}$, etc., lying more nearly longitudinally.

The extensor tendon attaches to the dorsal rim of the tibia by a broad ribbon-like portion and soon expands into a broad plate at right angles to this first portion and then
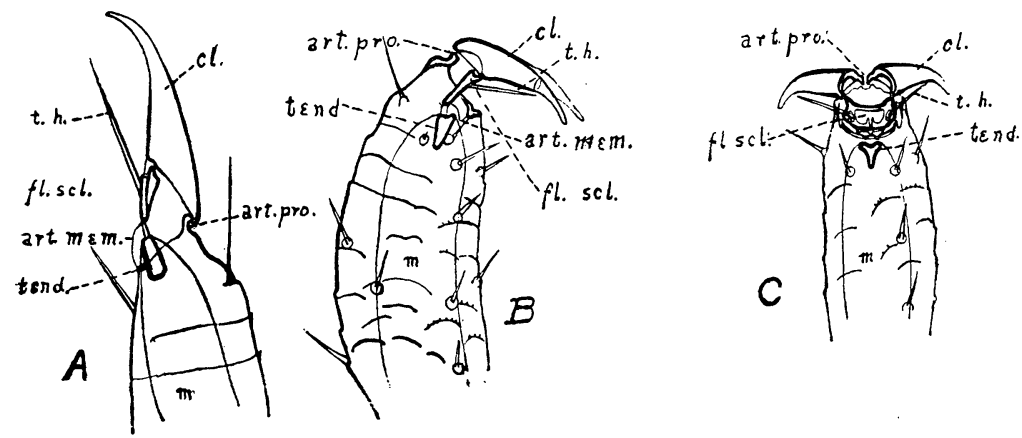

FIG. 2. End of foot of Aphis brassica. a, side view of claws at extreme extension; $b$, Ibid, claws flexed; $c$, viewed from beneath; $c l$, claw; $t . h$, tactile hair ; flscl, floating sclerite; art.pro, articular process ; art.mem, articular membrane; tend, tendon; $m$, muscle.

narrows to a ribbon and extends deeply into the femur even in the earliest stages. A short muscle, $e$, is attached to the disk, followed by a series of others, somewhat as the flexor muscles are arranged, only that there is but a single series, finding their attachment to the middle dorsal side of the femur. Tendons are first developed as somewhat tubular processes, but always collapse after the 
molt so that the tubular character can never be made out. In the case of the extensor tendon of the knee the enlarged disk must require a considerable stretching of the portion of the tendon further out to enable it to pass.

The tendon of the claw is very short up to the last molt in the case of plant lice. The structure at the end of the last tarsal joint is shown in Fig. 2. At the extreme end of the foot there are two processes over which the base of the claws rotate. The only other attachment aside from the soft articular membrane is a median floating sclerite capping the larger part of the end of the cavity of the foot and which bears the tactile spines extending forward below the claws. This floating sclerite in other insects forms the base of the empodium and pulvillæ. Neither of these is present in the case of the plant lice unless the soft skin immediately beyond this sclerite be so designated. The lower edge of the margin of the cavity is a strongly developed ridge upon which the internal tendon bears when the claws are extended, and against which the floating sclerite rests in extreme flexion. On either side of this thickened and elevated ridge there is a distinct notch allowing considerable lateral motion of the sclerite. The posterior ridge of this floating sclerite extends inward as two processes joining with the two wings of the heartshaped tendon. The tendon proper is entirely internal as is shown in the figure, and the muscle fibers are attached to all sides. The other attachment of the muscle is to the base of this large second joint of the tarsus.

There are really no true tendons in insects; $i$. $e$, the tendons of the leg's are only such in a physiological or morphological sense, and not at all in structure or origin, but belong instead to the class of internal processes which includes the well-known internal skeleton or the head and thorax, the tendons of the jaws in mandibulate insects, the great internal disk-like tendons for the attachment of the elevator muscles of the wings in the Odonata, and the skeletal and tendonal process of the ovipositor. The 
only difference between a skeletal process and a tendon is that one is invaginated from a relatively fixed part of the body and the other from a moving part. While insect tendons are, therefore, not homologous with the tendons of vertebrates, it is probably wise to retain the name just as in the case of femur and tibia for parts of the leg, where likewise there is no homology with the bones of vertebrates where the names primarily apply. 